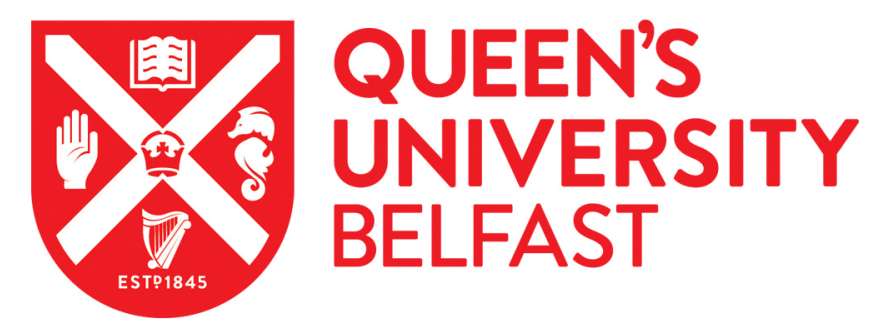

\title{
Northern Ireland - Republic of Ireland comparative data 2020
}

Whitten, L. (2021). Northern Ireland - Republic of Ireland comparative data 2020. Irish Political Studies, 36(3). https://doi.org/10.1080/07907184.2021.1938361

Published in:

Irish Political Studies

Document Version:

Publisher's PDF, also known as Version of record

Queen's University Belfast - Research Portal:

Link to publication record in Queen's University Belfast Research Portal

\section{Publisher rights}

Copyright 2021 the authors.

This is an open access article published under a Creative Commons Attribution-NonCommercial-NoDerivs License

(https://creativecommons.org/licenses/by-nc-nd/4.0/), which permits distribution and reproduction for non-commercial purposes, provided the author and source are cited.

\section{General rights}

Copyright for the publications made accessible via the Queen's University Belfast Research Portal is retained by the author(s) and / or other copyright owners and it is a condition of accessing these publications that users recognise and abide by the legal requirements associated with these rights.

Take down policy

The Research Portal is Queen's institutional repository that provides access to Queen's research output. Every effort has been made to ensure that content in the Research Portal does not infringe any person's rights, or applicable UK laws. If you discover content in the Research Portal that you believe breaches copyright or violates any law, please contact openaccess@qub.ac.uk. 


\section{Irish Political Studies}

\section{Northern Ireland - Republic of Ireland comparative data 2020}

\section{Lisa Whitten}

To cite this article: Lisa Whitten (2021): Northern Ireland - Republic of Ireland comparative data 2020, Irish Political Studies, DOI: 10.1080/07907184.2021.1938361

To link to this article: https://doi.org/10.1080/07907184.2021.1938361
(2) 2021 The Author(s). Published by Informa UK Limited, trading as Taylor \& Francis Group

\section{里 Published online: 05 Jul 2021.}

Submit your article to this journal $\widetilde{ }$

Q View related articles $₫$

View Crossmark data $\nearrow$ 


\section{Northern Ireland - Republic of Ireland comparative data 2020}

\section{Lisa Whitten}

School of History, Anthropology, Philosophy and Politics, Queen's University, Belfast, UK

\section{List of abbreviations}

1. North-South Relations

1.1. Cross-border Trade/Economic Activities

1.2. Bilateral, North-South Meetings

1.3. North-South Pandemic Statistics

2. UK-Irish Relations

2.1. Economic

2.2. Bilateral

2.3. Diplomatic

3. UK-Ireland Internationally

3.1. Comparative State of Democracy

3.2. Comparative State of Economy

3.3. Comparative Population Statistics

3.4. Comparative Pandemic Statistics

\section{List of abbreviations}

\begin{tabular}{ll}
\hline CSO & Central Statistics Office \\
GB & Great Britain \\
GDP & Gross Domestic Product \\
EIU & Economist Intelligence Unit \\
IMF & International Monetary Fund \\
ONS & Office for National Statistics \\
ROI & Republic of Ireland \\
RSF & Reporters Sans Frontières \\
SEUPB & Special EU Programmes Body \\
TI & Transparency International \\
UK & United Kingdom \\
\hline
\end{tabular}

CONTACT Lisa Whitten whitten02@qub.ac.uk

() 2021 The Author(s). Published by Informa UK Limited, trading as Taylor \& Francis Group

This is an Open Access article distributed under the terms of the Creative Commons Attribution-NonCommercial-NoDerivatives License (http://creativecommons.org/licenses/by-nc-nd/4.0/), which permits non-commercial re-use, distribution, and reproduction in any medium, provided the original work is properly cited, and is not altered, transformed, or built upon in any way. 


\section{North-South relations}

\subsection{Cross border trade/economic activities}

Table 1.1.1. Cross-border trade in goods and services (all figures in billion $€$ )

\begin{tabular}{lllllr}
\hline & 2014 & 2015 & 2016 & 2017 & $2018^{\mathrm{a}}$ \\
\hline Goods & 1.75 & 1.83 & 1.63 & 3.81 & 3.90 \\
Services & 3.32 & 3.27 & 2.98 & 3.19 & 3.48 \\
Total & 5.07 & 5.1 & 4.61 & 7.0 & 7.38 \\
\hline
\end{tabular}

Source: InterTrade Ireland https://bit.ly/2P4uYdJ.

atatest available [Requested].

\subsection{Bilateral North-South meetings}

Table 1.2.1. Meetings of North-South bodies established under the Belfast 'Good Friday' Agreement.

\begin{tabular}{lll}
\hline $\begin{array}{l}\text { North-South Ministerial } \\
\text { Council }\end{array}$ & $\begin{array}{c}\text { North-South Inter-Parliamentary } \\
\text { Association }\end{array}$ & $\begin{array}{c}\text { North-South Consultative } \\
\text { Forum }\end{array}$ \\
\hline $\begin{array}{c}\text { 11th Institutional Meeting on } \\
11 \text { March 2020 }\end{array}$ & $\begin{array}{c}\text { Has not met since 2 December } \\
\text { 2016 due to the three-year } \\
\text { hiatus in operation of the } \\
\text { 24th Plenary on 31 July 2020 } \\
\text { in Dublin }\end{array}$ & $\begin{array}{c}\text { Has never been formally } \\
\text { established or convened } \\
\text { despite commitments to do so } \\
\text { in both the Good Friday and }\end{array}$ \\
$\begin{array}{c}\text { 12th Institutional Meeting on } \\
\text { 12 March 2020 }\end{array}$ & $\begin{array}{l}\text { (January 2017-20) followed by } \\
\text { the outbreak of the coronavirus }\end{array}$ & $\begin{array}{l}\text { St. Andrews Agreements } \\
\text { pandemic }\end{array}$ \\
$\begin{array}{l}\text { 25th Plenary on 18 December } \\
\text { 2020 in Armagh }\end{array}$ & \\
$\begin{array}{l}\text { Source: North South Ministerial Council, https://bit.ly/3tyfCNf; North South Inter-Parliamentary Associ- } \\
\text { ation, https://bit.ly/2TcJYo9; on North South Consultative Forum see Houses of the Oireachtas state- }\end{array}$ \\
ment, https://bit.ly/312wAqN.
\end{tabular}

Table 1.2.2. Meetings of North-South Implementation Bodies established under the Belfast 'Good Friday' agreement.

\begin{tabular}{ll}
\hline Area of cooperation & Meeting held \\
\hline Health and Food Safety Body & 2 October \\
Transport & 7 October \\
Environment & 21 October \\
Aquaculture and Marine & 21 October \\
SEUPB & 30 October \\
Education & 6 November \\
Inland Waterways & 11 November \\
Agriculture & 18 November \\
Trade and Business Development & 25 November \\
Tourism & 25 November \\
Language Body & 27 November \\
\hline
\end{tabular}

Source: North South Ministerial Council, https://bit.ly/38Q54Bp. 


\subsection{North-South pandemic statistics}

Table 1.3.1. North-South pandemic statistics

\begin{tabular}{lll}
\hline & Northern Ireland & Ireland \\
\hline Total Cases & 74,677 & 91,779 \\
Total Deaths & $1,903^{\mathrm{a}}$ & 2,226 \\
\hline
\end{tabular}

Source: Coronavirus Data UK Government, https://bit.ly/2009dyV; Covid-19 Data Irish Government, https://bit.ly/38ZNGKr.

${ }^{a}$ no. with COVID-19 on death certificate.

\section{UK-Irish relations}

\subsection{Economic activities}

Table 2.1.1. 2020 import and export of goods between Ireland and Great Britain by month (All figures are in millions $€$ ).

Table 2.1.2. 2019-2020 import and export of services between Ireland and the UK (All figures are in millions $€$ ).

\begin{tabular}{lll}
\hline Month & Irish imports from GB & Irish exports to GB \\
\hline January & 1,496 & 1,142 \\
February & 1,477 & 1,021 \\
March & 1,607 & 1,047 \\
April & 1,439 & 865 \\
May & 1,371 & 1,107 \\
June & 1,449 & 897 \\
July & 1,535 & 988 \\
August & 1,325 & 1,072 \\
September & 1,451 & 1,072 \\
October & 2,045 & 1,041 \\
November & 1,879 & 1,459 \\
December $^{\text {2020 Total }}$ & 1,606 & 1,084 \\
\hline
\end{tabular}

Source: CSO Trade Statistics, https://bit.ly/2NwAWUf

${ }^{\mathrm{a}}$ To the nearest million $€$.

\begin{tabular}{lll}
\hline & Irish imports from UK & Irish exports to UK \\
\hline 2019 Quarter 1 & 4,711 & 7,798 \\
Quarter 2 & 5,047 & 8,504 \\
Quarter 3 & 5,137 & 8,972 \\
Quarter 4 & 5,648 & 9,622 \\
2020 Quarter 1 & 4,952 & 8,271 \\
Quarter 2 & 4,357 & 7,787 \\
Quarter 3 & 4,371 & 8,518 \\
\hline
\end{tabular}

Source: CSO Trade Statistics, https://bit.ly/3vHF8I2 
Table 2.1.3. 2019-2020 import and export of merchandise between Ireland and the UK (All figures are in millions $€$ ).

\begin{tabular}{lll}
\hline & Irish imports from UK & Irish exports to UK \\
\hline 2019 Quarter 1 & 6,895 & 5,140 \\
Quarter 2 & 5,907 & 5,171 \\
Quarter 3 & 6,209 & 5,261 \\
Quarter 4 & 7,030 & 5,704 \\
2020 Quarter 1 & 6,103 & 4,986 \\
Quarter 2 & 5,674 & 4,448 \\
Quarter 3 & 5,852 & 4,916 \\
\hline
\end{tabular}

Source: CSO Trade Statistics, https://bit.ly/3vHF812

\subsection{Bilateral meetings}

Table 2.2.1. Meetings of East-West bodies established under the Good Friday Agreement

\begin{tabular}{lcc}
\hline $\begin{array}{l}\text { British-Irish } \\
\text { Intergovernmental } \\
\text { Conference }\end{array}$ & \multicolumn{1}{c}{ British-Irish Council } & $\begin{array}{c}\text { British-Irish Parliamentary } \\
\text { Assembly }\end{array}$ \\
\hline $\begin{array}{l}\text { No meetings held due to } \\
\text { coronavirus pandemic }\end{array}$ & $\begin{array}{l}\text { Only summit of the year (34th) was } \\
\text { hosted by Scottish Government virtually } \\
\text { on 6 November }\end{array}$ & $\begin{array}{c}\text { No meetings held due to } \\
\text { coronavirus pandemic. }\end{array}$ \\
\hline
\end{tabular}

Source: British-Irish Intergovernmental Conference, https://bit.ly/3|Bo5N1; British-Irish Council, https:// bit.ly/2OLHsHe; British-Irish Inter-Parliamentary Assembly, https://bit.ly/3trK8IO.

Table 2.2.2. Meetings between British Prime Minister and Irish Taoiseach ${ }^{a}$.

\begin{tabular}{lcc}
\hline Location & Date & \multicolumn{1}{c}{ Event } \\
\hline Virtual & 30 June & $\begin{array}{c}\text { British Prime Minister Johnson congratulates Irish Taoiseach Micheál Martin } \\
\text { on his recent appointment and discusses bilateral relations and shared } \\
\text { interests in telephone call }\end{array}$ \\
Belfast & 13 August & $\begin{array}{c}\text { Meeting to discuss bilateral relations, Northern Ireland, UK-EU relations and } \\
\text { shared positions on global issues } \\
\text { Conversation to discuss Ireland's decision to enter Level } 5 \text { lockdown; British } \\
\text { Prime Minister expresses concern about creating a large disparity between } \\
\text { guidelines on either side of land border. }\end{array}$ \\
Virtual & 8 October & $\begin{array}{c}\text { Meeting to discuss UK-EU trade negotiations and reaffirm joint commitment } \\
\text { to prioritize Belfast 'Good Friday' Agreement and avoid hard land border in } \\
\text { context of UK leaving EU }\end{array}$ \\
\hline
\end{tabular}

Sources: Gov.UK; The Irish Times; Reuters.

ancludes virtual meetings in view of travel restrictions caused by coronavirus pandemic.

\subsection{UK-Ireland diplomatic relations}

Table 2.3.1. Diplomatic meetings ${ }^{\mathrm{a}}$

\begin{tabular}{llc}
\hline & \multicolumn{1}{c}{ Location } & Date \\
\hline Princess Royal & Dublin & 1 February \\
Duke and Duchess of Cambridge & Dublin and Galway & $3-5$ March
\end{tabular}


Continued.

\begin{tabular}{lll}
\hline & Location & Date \\
\hline Prince of Wales w/President of Ireland & Telephone & 1 October \\
Prince of Wales w/President of Ireland & Telephone & 24 November \\
\hline
\end{tabular}

Source: President of Ireland Engagement Diary, https://bit.ly/2whzWu0; and Court Circular, https://bit.ly/ 37Dg41G.

ancludes virtual meetings in view of travel restrictions caused by coronavirus pandemic.

\section{UK-Ireland internationally}

\subsection{Comparative state of democracy}

\section{Table 3.1.1. Democracy ratings}

\begin{tabular}{|c|c|c|c|c|}
\hline \multirow{2}{*}{$\frac{\text { Country }}{\text { Ireland }}$} & \multicolumn{2}{|c|}{ EIU Democracy Index $( \pm)$} & \multicolumn{2}{|c|}{ Freedom House $( \pm)$} \\
\hline & $8^{\text {th }}(-)$ & $9.05(-0.19)$ & Free $(-)$ & $97(-)$ \\
\hline United Kingdom & $16^{\text {th }}(-)$ & $8.54(+0.02)$ & Free $(-)$ & $93(-1)$ \\
\hline
\end{tabular}

Note: Max score is 10 for EIU Democracy Index and 100 for Freedom House. Change in scores and rankings are in comparison with previous year. Source: The Economist, https://bit.ly/3sf3cKh; and Freedom House, https://bit.ly/2OREgd0.

Table 3.1.2. Press freedom index

\begin{tabular}{lllll}
\hline Country & \multicolumn{2}{c}{ RSF Press Freedom Index $( \pm)$} & \multicolumn{2}{c}{ TI Corruption Index $( \pm)$} \\
\hline Ireland & 13th $(+2)$ & $12.6(-2.4)$ & 20th $(-2)$ & $72(-2)$ \\
United Kingdom & 35th (-2) & $22.93(+0.7)$ & 11 th $(+1)$ & $77(-)$ \\
\hline
\end{tabular}

Note: Change in scores and rankings are in comparison with previous year. Source: Transparency International, https://bit.ly/3vBljuR; Reporters Sans Frontières, https://bit.ly/2PbGAva.

\subsection{Comparative state of economy}

Table 3.2.1. GDP growth

\begin{tabular}{lcl}
\hline Country & GDP Growth & GDP Total in US\$ (million) \\
\hline Ireland & $4.9 \%$ & $388,698.71$ \\
United Kingdom & $5.9 \%$ & $2,829,108.22$ \\
\hline
\end{tabular}

Source: IMF World Economic Outlook (October 2020), https://bit.ly/3lxr2hF; and World Bank Data (2019), https://bit.ly/20S72u2.

aLatest available. 
Table 3.2.2. Public debt

\begin{tabular}{lc}
\hline Country & General Government Gross Debt as \% of GDP \\
\hline Ireland & 61.3 \\
United Kingdom & 111.5
\end{tabular}

Source: IMF World Economic Outlook (October 2020), https://bit.ly/2P6yu7p.

\section{Table 3.2.3. Private debt}

\begin{tabular}{lc}
\hline Country & Household debt, loans and securities as \% of GDP \\
\hline Ireland & 37.56 \\
United Kingdom & 83.99 \\
\hline
\end{tabular}

Source: IMF World Economic Outlook (October 2020), https://bit.ly/2P6yu7p.

Table 3.2.4. Comparative import and export destinations

\begin{tabular}{|c|c|c|c|c|}
\hline Country & \multicolumn{2}{|c|}{ Export market (\% Share) } & \multicolumn{2}{|c|}{ Import market (\% Share) } \\
\hline \multicolumn{5}{|l|}{ Ireland } \\
\hline & United States & 31 & United Kingdom & 22 \\
\hline & Belgium & 11.1 & United States & 14.5 \\
\hline & Germany & 10.8 & France & 11 \\
\hline & United Kingdom & 8.99 & Germany & 7.89 \\
\hline & China & 6.12 & China & 7.14 \\
\hline \multicolumn{5}{|c|}{ United Kingdom } \\
\hline & United States & 14.4 & China & 11.9 \\
\hline & Germany & 10.3 & Germany & 11.6 \\
\hline & Ireland & 6.83 & United States & 9.22 \\
\hline & Netherlands & 6.2 & Netherlands & 7.21 \\
\hline & France & 5.88 & France & 4.64 \\
\hline
\end{tabular}

Source: Trend Economy (2020), https://bit.ly/3eWCFxl.

\subsection{Comparative population statistics}

Table 3.3.1. Total population

\begin{tabular}{lc}
\hline Country & Population (million) \\
\hline Ireland & 5.01 \\
United Kingdom & 67.26
\end{tabular}

Source: IMF World Economic Outlook (December 2020), https://bit.ly/2OFqJW9.

\section{Table 3.3.2. Migration figures}

\begin{tabular}{llll}
\hline Country & Net migration & Immigration & Emigration \\
\hline Ireland & 28,900 & 85,400 & 56,500 \\
United Kingdom & 313,000 & 715,000 & 403,000 \\
\hline
\end{tabular}

Source: Office for National Statistics, https://bit.ly/3cS7Tmq; and Central Statistics Office, https://bit.ly/ 38W0I13. Note: UK figures for calendar year ending March 2020 (ONS, August 2020); ROI figures for year ending April 2020 (CSO, August 2020). 


\subsection{Comparative pandemic statistics}

Table 3.4.1. Comparative total COVID-19 cases and deaths

\begin{tabular}{lcr}
\hline Country & \multicolumn{1}{c}{ Cases } & Deaths \\
\hline Ireland & 91,779 & 2,226 \\
United Kingdom & $2,657,358$ & $94,627^{\mathrm{a}}$ \\
\hline Source: Coronavirus Data UK Government, https://bit.ly/2009dyV; Covid-19 Data Irish Government, \\
https://bit.ly/38ZNGKr. & & \\
ano. with COVID-19 on death certificate. & &
\end{tabular}

\section{Disclosure statement}

No potential conflict of interest was reported by the author(s). 